\title{
Das Verhältnis des nationalen Rechts zum Europarecht gemäß der Rechtsprechung des Europäischen Gerichtshofes mit Beispielen aus dem Arbeits- und Sozialrecht*
}

\author{
Vassilios SKOURIS
}

Die Europäische Gemeinschaft nimmt unter den Formen zwischenstaatlicher $\mathrm{Zu}-$ sammenarbeit eine Sonderstellung ein. Diese Sonderstellung ergibt sich insbesondere aus ihrem supranationalen Charakter und sie zeigt sich nicht zuletzt auch an der Eigenart des von ihr geschaffenen Rechts.

Das Gemeinschaftsrecht beansprucht Vorrang vor dem Recht der Mitgliedstaaten und setzt sich im Kollisionsfalle gegenüber dem nationalen Recht durch; es lässt nationales Recht dabei zwar nicht ungültig werden, verhindert aber dessen Anwendung, soweit die Kollision reicht. Aufgrund der unmittelbaren Anwendbarkeit des Gemeinschaftsrechts kann sich der Einzelne vor Behörden und Gerichten auf Gemeinschaftsrecht berufen und die Behörden und die Gerichte sind berechtigt - aber auch verpflichtet - Gemeinschaftsrecht anzuwenden.

Vorrang und unmittelbare Anwendbarkeit gehören $\mathrm{zu}$ den wichtigsten Rechtsgrundsätzen des Gemeinschaftsrechts. Sie haben starke Wurzeln, indem sie auf die berühmten Urteile Costa/E.N.E.L. ${ }^{1}$ und Van Gend \& Loos $^{2}$ des Europäischen Gerichtshofes aus den sechziger Jahren des letzten Jahrhunderts zurückgehen. Diese Grundsätze, oder auch ,Verfassungs“"-Prinzipien, sind seither fester Bestandteil des Gemeinschaftsrechts und bestimmen das Verhältnis des nationalen Rechts zum Gemeinschaftsrecht. Sie gehören zum acquis communautaire, den alle seither beigetretenen Mitgliedstaaten übernommen haben. Im Folgenden möchte ich auf beide Grundsätze etwas näher eingehen.

\section{Unmittelbare Wirkung des Gemeinschaftsrechts}

In der Entscheidung Van Gend \& Loos aus dem Jahre 1963 hat der Europäische Gerichtshof erstmals ausdrücklich zur Frage der unmittelbaren Wirkung des Gemein-

* Vortrag im Rahmen des von der deutschen Ratspräsidentschaft veranstalteten Symposiums „50 Jahre EU - 50 Jahre Rechtsprechung des Europäischen Gerichtshofs zum Arbeits- und Sozialrecht" am 25. Juni 2007 in Berlin. - Für die Veröffentlichung wurde der Text, unter Beibehaltung der Vortragsform, durchgesehen und um Nachweise ergänzt.

1 EuGH, 6/64, Slg. 1964, 1251 - Costa/E.N.E.L.

2 EuGH, 26/62, Slg. 1963, 1 - Van Gend \& Loos. 
schaftsrechts Stellung genommen. Der EG-Vertrag richte sich „,nicht nur an die Regierungen, sondern auch an die Völker“. Die Gemeinschaft schaffe „eine neue Rechtsordnung des Völkerrechts, [...] deren Rechtssubjekte nicht nur die Mitgliedstaaten, sondern auch die Einzelnen sind“. Das Gemeinschaftsrecht solle „daher den Einzelnen, ebenso wie es ihnen Pflichten auferlegt, auch Rechte verleihen“. Aufgrund dessen entfaltet das Gemeinschaftsrecht eine unmittelbare Wirkung mit der Folge, dass sich natürliche und juristische Personen auf die ihnen durch den Vertrag verliehenen Rechte berufen können $^{3}$.

Unmittelbar anwendbar sind demgemäß alle diejenigen Bestimmungen des EGVertrages, die klar und unbedingt sind und keiner mitgliedstaatlichen Umsetzung bedürfen. Für eine Vielzahl von Vertragsbestimmungen, insbesondere für die Grundfreiheiten des Binnenmarktes oder den sozialpolitischen und arbeitsrechtlichen Grundsatz des gleichen Entgelts für Männer und Frauen bei gleicher oder gleichwertiger Arbeit hat der Europäische Gerichtshof dies angenommen ${ }^{4}$. Für Verordnungen ergibt sich die unmittelbare Anwendbarkeit schon aus ihrer Definition im EG-Vertrag 5 . Bei Richtlinien liegen die Dinge etwas komplizierter. Sie sind zwar an die Mitgliedstaaten gerichtet; jedoch kann eine Richtlinienbestimmung nach der Rechtsprechung dann unmittelbar angewendet werden, wenn sie inhaltlich unbedingt und hinreichend genau gefasst sind. Ist das der Fall, so kann sich der Einzelne bei fehlender oder unzulänglicher Umsetzung der Richtlinienbestimmung vor den nationalen Behörden und Gerichten auf sie berufen ${ }^{6}$.

Mit dieser Rechtsprechung wird dem Staat die Möglichkeit genommen, sich sein eigenes Versäumnis bei der Richtlinienumsetzung zunutze zu machen und dem Einzelnen Rechte zu verwehren, die dieser bei rechtzeitiger und korrekter Umsetzung haben würde. Dieser Sanktionsaspekt ist einer der Gründe, warum die unmittelbare Anwendbarkeit oder Wirkung nicht grenzenlos sein kann. Denn er macht keinen Sinn gegenüber jemandem, der für die fehlende oder fehlerhafte Umsetzung nicht verantwortlich ist bzw. nicht verantwortlich gemacht werden kann. Wenn die nicht oder nicht korrekt umgesetzte Richtlinienbestimmung eine Verpflichtung für einen Einzelnen betrifft, kann sich daher weder eine andere private Person noch der Staat selbst auf die betreffende Richtlinienbestimmung berufen, mag sie noch so klar und bestimmt sein. Die unmittelbare Anwendbarkeit einer Richtlinienbestimmung kann somit nur zu Lasten des Staates, aber nicht zu Lasten eines Bürgers gehen.

Auf das Arbeits- und Sozialrecht bezogen heißt das, dass die unmittelbare Anwendbarkeit einer Richtlinienbestimmung im Sozialrecht häufiger als im Arbeitsrecht in Fra-

3 EuGH, 26/62, Slg. 1963, 3 (24/25) - Van Gend \& Loos.

4 Nachweise bei Skouris/Kraus, Die Bedeutung der Grundfreiheiten und Grundrechte für das europäische Wettbewerbsrecht, in: Münchner Kommentar zum europäischen Wettbewerbsrecht, Band 1, 2007 (im Erscheinen), Rz. 283.

5 Art. 249 Abs. 2 EGV.

6 Bspw. EuGH, C-6/90 und C-9/90, Slg. 1991, I-5357, Rz. 11 - Francovich e.a.; EuGH, C-62/00, Slg. 2002, I-6325, Rz. 25 - Marks \& Spencer; EuGH, C-397/01 bis C-403/01, Slg. 2004, I-8835, Rz. 103 - Pfeiffer e.a. 
ge kommt. Denn während sich in sozialrechtlichen Streitigkeiten typischerweise Staat und Bürger gegenüberstehen, sind es im Individualarbeitsrecht häufig zwei private Parteien, mit der Folge, dass mit der Verbesserung der Rechtsstellung der einen privaten Seite eine Verschlechterung für die andere private Seite korrespondieren wird.

Die Rechtssache Pfeiffer spiegelt diesen Umstand wider. Dort ging es um einen Rechtsstreit zwischen dem Deutschen Roten Kreuz und einigen Rettungsassistenten über die Frage, ob Deutschland die Arbeitszeitrichtlinie im Hinblick auf die zulässigen Höchstarbeitszeiten korrekt umgesetzt hat ${ }^{7}$. Da die unmittelbare Anwendung einer Richtlinie nicht zu Lasten eines Bürgers gehen darf, war es ausgeschlossen, auf die Frage des Arbeitsgerichts Lörrach zu antworten, dass die Richtlinie unmittelbar anzuwenden sei. Der Europäische Gerichtshof gab jedoch dem vorlegenden Gericht auf, genau zu prüfen, inwieweit das nationale Recht richtlinienkonform ausgelegt werden kann, um wenn irgend möglich zu dem von der Richtlinie geforderten Ergebnis zu kommen, ohne dabei die Grenzen zulässiger Auslegung zu überschreiten ${ }^{8}$. Zu diesen Grenzen gehört insbesondere die Wortlautgrenze; eine Auslegung nationalen Rechts contra legem ist daher unzulässig 9 .

\section{Der Vorrang des Gemeinschaftsrechts}

Auch die Frage, ob das Gemeinschaftsrecht im Falle der Kollision Vorrang vor dem nationalen Recht beanspruchen kann, wurde bereits früh in der Rechtsprechung des Europäischen Gerichtshofes behandelt.

Im 1964 ergangenen Urteil Costa/E.N.E.L. führte der Europäische Gerichtshof aus, dass die Gemeinschaftsrechtsordnung zwar völkervertragsrechtlich zwischen den Mitgliedstaaten begründet worden ist, sie aber nunmehr als autonome Rechtsordnung bestehe. Die somit von der zwischenstaatlichen Gegenseitigkeit abgekoppelten Rechte und Pflichten dürfen nicht einseitig durch später ergehende innerstaatliche Maßnahmen der Mitgliedstaaten beeinträchtigt werden. Die Regel „lex posterior derogat legi priori“ gilt daher im Verhältnis zwischen nationalem Recht und Gemeinschaftsrecht nicht. Wörtlich heißt es in dem Urteil Costa/E.N.E.L.: „Aus alledem folgt, dass dem vom Vertrag geschaffenen, somit aus einer autonomen Rechtsquelle fließenden Recht wegen dieser seiner Eigenständigkeit keine wie immer gearteten innerstaatlichen Rechtsvorschriften vorgehen können, wenn ihm nicht sein Charakter als Gemeinschaftsrecht aberkannt und

7 EuGH, C-397/01 bis C-403/01, Slg. 2004, I-8835 - Pfeiffer e.a.

8 Zur richtlinienkonformen Auslegung siehe $V$. Skouris, Rechtswirkungen von nicht umgesetzten EGRichtlinien und EU-Rahmenbeschlüssen gegenüber Privaten - neuere Entwicklungen in der Rechtsprechung des EuGH, ZEuS 2005, $463 \mathrm{ff}$.

9 EuGH, C-212/04, Slg. 2006, I-6057, Rz. 110 - Adeneler e.a. 
wenn nicht die Rechtsgrundlage der Gemeinschaft selbst in Frage gestellt werden soll." $" 10$

Der Vorrang wird also nicht vom nationalen Recht und den jeweiligen Ermächtigungsnormen in den nationalen Rechtsordnungen her begründet. Vielmehr wird er als gemeinschaftsrechtlich notwendig angesehen, um die Funktionsfähigkeit der Gemeinschaft innerhalb der Grenzen ihrer Zuständigkeiten zu sichern.

Die mitgliedstaatlichen Gerichte sind daher gehalten, für die volle Wirksamkeit des Gemeinschaftsrechts Sorge zu tragen, indem sie erforderlichenfalls jede - auch spätere - entgegenstehende Bestimmung des nationalen Rechts aus eigener Entscheidungsbefugnis unangewendet lassen. Um dies tun zu können, brauchen sie nicht die vorherige Beseitigung dieser Bestimmung auf gesetzgeberischem Wege oder anderem Wege abzuwarten ${ }^{11}$. Das gilt gegebenenfalls auch für nationale Verfassungsbestimmungen, wie der Europäische Gerichtshof in einer Entscheidung Anfang der siebziger Jahre präzisierte ${ }^{12}$.

In diesem Zusammenhang möchte ich an die Rechtssache Kreil erinnern, welche die Frage betraf, ob es mit dem Gemeinschaftsrecht vereinbar ist, dass in der deutschen Bundeswehr sämtliche bewaffneten Einheiten ausschließlich aus Männern bestehen mussten ${ }^{13}$. Der damalige Art. 12a des Grundgesetzes verbot, dass Frauen Dienst mit der Waffe leisten ${ }^{14}$. Für Frau Kreil bedeutete das, dass sie nicht als Elektronikerin in der Bundeswehr arbeiten konnte. Ihre Bewerbung wurde daher abgelehnt. Als sie dagegen klagte, wurde der Europäische Gerichtshof im Wege der Vorabentscheidung mit der Frage befasst und kam zu dem Ergebnis, dass eine nationale Regelung, die Frauen allgemein vom Dienst mit der Waffe ausschließt und ihnen nur den Zugang zum Sanitätsund Militärmusikdienst erlaubt, mit der Richtlinie über den gleichen Zugang von Männern und Frauen zur Beschäftigung unvereinbar ist. Die Bundesrepublik Deutschland hat daraufhin das Grundgesetz geändert. Die betreffende Verfassungsbestimmung sieht nunmehr vor, dass Frauen auf keinen Fall zum Dienst mit der Waffe verpflichtet werden dürfen 15 .

Der Grundsatz des Vorrangs wird auch bei der Auslegung und Anwendung der Bestimmungen des Gemeinschaftsrechts relevant. Die nationalen Gerichte haben ihrer

10 EuGH, 6/64, Slg. 1964, 1253 (1270) - Costa/E.N.E.L.

11 EuGH, 106/77, Slg. 1978, S. 629 - Simmenthal.

12 EuGH, 11/70, Slg. 1970, S. 1125 - Internationale Handelgesellschaft: Die Gültigkeit von Gemeinschaftsrecht könne auch dann nicht in Frage gestellt werden, wenn geltend gemacht wird, dass Grundrechte oder grundlegende Strukturprinzipien der nationalen Verfassung verletzt seien. Prüfungsmaßstab seien in solchen Situationen nicht die allgemeinen Grundsätze des nationalen Rechts, sondern diejenigen des Gemeinschaftsrechts, einschließlich der Gemeinschaftsgrundrechte.

13 EuGH, C-285/98, Slg. 2000, I-69 - Kreil.

14 Art. 12a Abs. 4 Satz 2 GG a.F.: „Sie dürfen auf keinen Fall Dienst mit der Waffe leisten.“

15 Art. 12a Abs. 4 Satz 2 GG in der Fassung des Gesetzes zur Änderung des Grundgesetzes (Artikel 12a) vom 19.12.2000 (BGBl. I S. 1755): „Sie dürfen auf keinen Fall zum Dienst mit der Waffe verpflichtet werden.“ 
Rechtsprechung diejenige Auslegung des Gemeinschaftsrechts zugrunde zu legen, welche der Europäische Gerichtshof diesen Bestimmungen gegeben hat. Wenn sich im Zuge eines Vorabentscheidungsverfahrens ergeben sollte, dass eine gemeinschaftsrechtliche Vorschrift auf nationaler Ebene bislang in unzutreffender Weise ausgelegt wurde, dann ist dies dort zu korrigieren, auch wenn das die Aufgabe einer langjährigen höchstrichterlichen Rechtsprechung bedeutet. Denn durch die Auslegung einer gemeinschaftsrechtlichen Vorschrift durch den Europäischen Gerichtshof im Vorabentscheidungswege wird nur klargestellt, in welchem Sinne und mit welcher Tragweite diese Vorschrift seit ihrem Inkrafttreten zu verstehen ist oder gewesen wäre. Daher muss diese Auslegung grundsätzlich auch auf Rechtsverhältnisse, die vor Erlass des auf das Ersuchen um Auslegung ergangenen Urteils entstanden sind, gelten ${ }^{16}$.

Die Richtlinie zur Massenentlassung 17 bietet ein aktuelles Beispiel für das Zusammenspiel von nationalen Gerichten und Europäischem Gerichtshof in dieser Hinsicht.

Das Bundesarbeitsgericht hatte den Begriff der „Entlassung“ bisher so verstanden, dass er nicht den Zeitpunkt des Ausspruchs der Kündigung, sondern deren Wirksamwerden meint ${ }^{18}$. Da zwischen Ausspruch der Kündigung und ihrem Wirksamwerden eine beträchtliche Zeitspanne liegen kann, schon wegen der Kündigungsfristen, kommt diesem Aspekt eine große Bedeutung zu. In der Rechtssache Junk entschied der Europäische Gerichtshof auf Vorlage des Arbeitsgerichts Berlin jedoch, dass mit dem Begriff „Entlassung“ im Sinne der Richtlinie der Zeitpunkt des Ausspruchs der Kündigung gemeint ist. Denn die Konsultations- und Anzeigepflichten, die gemäß der Richtlinie vor der Entlassung zu beachten sind und die dem Ziel dienen, Kündigungen zu vermeiden oder ihre Zahl zu verringern, verlören ihren Sinn, wenn sie erst nach dem Ausspruch der Kündigung erfolgen könnten ${ }^{19}$. Das Bundesarbeitsgericht hat daraufhin seine Rechtsprechung geändert und legt nun das deutsche, der Umsetzung der Massenentlassungsrichtlinie dienende Recht richtlinienkonform aus, so dass der Begriff der Entlassung im nationalen Recht mit demjenigen der Richtlinie übereinstimmt ${ }^{20}$.

\section{Nationales und Gemeinschaftsrecht als ,europäische Rechtsordnung“}

Ohne weiter auf die schwierigen und technischen Einzelheiten dieses Beispiels aus dem Arbeitsrecht eingehen zu wollen, möchte ich am Schluss noch einen allgemeineren

16 EuGH, C-453/00, Slg. 2004, I-837, Rz. 21, 22 - Kühne \& Heitz; EuGH, C-292/04, Urteil vom 6.3.2007, Rz. 34 - Meilicke.

17 Richtlinie 98/59/EG des Rates vom 20. Juli 1998 zur Angleichung der Rechtsvorschriften der Mitgliedstaaten über Massenentlassungen (ABl. L 225, S. 16).

18 Nachweise im Urteil des BAG vom 18.9.2003, BAGE 107, $318=$ AP Nr. 14 zu $\S 17$ KSchG 1969.

19 EuGH, C-188/03, Slg. 2005, I-885, Rz. 31 ff. - Junk.

20 BAG, Urteil vom 23.3.2006 - 2 AZR 343/05 (Abdruck in BAGE vorgesehen), AP Nr. 21 zu $§ 17$ KSchG 1969, mit Anm. K. Riesenhuber. 
Aspekt hervorheben, der bei den von mir angeführten Beispielen schon angeklungen ist. Das Verhältnis von nationalem Recht und Gemeinschaftsrecht lässt sich nämlich nicht auf die Prinzipien der unmittelbaren Anwendbarkeit und des Vorrangs des Gemeinschaftsrechts oder gar auf die Rede von einem hierarchischen Verhältnis von nationalen und Gemeinschaftsgerichten reduzieren. Vielmehr können nationales Recht und Gemeinschaftsrecht nur zusammen die europäische Rechtsordnung schaffen und ausgestalten. Gleiches gilt für die mit der Kontrolle der Rechtsanwendung befassten Gerichte. Es ist daher auch kein Spiel mit Worten, wenn der Europäische Gerichtshof wiederholt zum Ausdruck gebracht hat, dass er sich nicht als allein für die Auslegung und Anwendung des Gemeinschaftsrechts zuständig ansieht, sondern diese Aufgabe - ich möchte fast sagen: zuallererst - den mitgliedstaatlichen Gerichten zukommt. Daraus erklärt sich auch die Rede von dem nationalen Richter als dem ,juge communautaire de droit commun" - eine Bezeichnung, die sagen möchte, dass die nationalen Richter Gemeinschaftsrichter sind, und zwar mit einer generellen bzw. umfassenden Zuständigkeit. Ohne eine aktive und sich ihrer Verantwortung bewusste Mitwirkung der mitgliedstaatlichen Gerichte kann das Gemeinschaftsrecht nicht zur Entfaltung kommen.

Daher kann die Aufgabe, mit den Mitteln des Rechts und der Rechtsprechung zum Gelingen der europäischen Integration beizutragen, nur im Wege vertrauensvollen $\mathrm{Zu}-$ sammenwirkens und wechselseitigen Respekts der nationalen Gerichte und des Europäischen Gerichtshofes gelingen. 


\title{
Zum Verhältnis des nationalen Rechts zum Europarecht nach der Rechtsprechung des Bundesverfassungsgerichts
}

\author{
Hans-Jürgen PAPIER
}

\section{Einführung}

Die Anwendung europäischen Rechts ist aus unserer täglichen Rechtspraxis kaum hinweg zu denken. In der Konsequenz stellen sich vielfältige Fragen des Verhältnisses von nationalem Recht zum Europarecht. Zu dem Symposium „50 Jahre EU - 50 Jahre Rechtsprechung des Europäischen Gerichtshofs zum Arbeits- und Sozialrecht" haben Sie mich eingeladen, um dieses Thema aus der Sicht des Bundesverfassungsgerichts und damit des deutschen Verfassungsrechts - zu beleuchten. Dies tue ich sehr gern. Wegen der nur begrenzt zur Verfügung stehenden Zeit muss ich mich allerdings auf eine knappe Darstellung aus der Sicht des Bundesverfassungsgerichts besonders wichtiger Fragestellungen beschränken. Hier rückt zum einen der Grundrechtsschutz gegen sekundäres Gemeinschaftsrecht und zum anderen die Kontrolle der Kompetenzgrenzen der Gemeinschaft in den Blick.

\section{Grundrechtsschutz gegen sekundäres Gemeinschaftsrecht}

Lassen Sie mich zunächst in groben Strichen die Entwicklung der Rechtsprechung des Bundesverfassungsgerichts zu den Problemen des Grundrechtsschutzes nachzeichnen. Den Ausgangspunkt bildete der Solange I-Beschluss aus dem Jahre 19741. Bereits zehn Jahre zuvor, in der Rechtssache Costa/E.N.E.L. ${ }^{2}$, hatte der EuGH die Grundsätze der unmittelbaren Geltung und des Vorrangs des Gemeinschaftsrechts als einer eigenständigen Rechtsordnung entwickelt; erst in Ansätzen erkennbar war auf der anderen Seite die Herausbildung eines begrenzenden Gegengewichts zur Rechtsetzung der Gemeinschaft, insbesondere durch grundrechtliche Gewährleistungen. Vor diesem Hintergrund hat sich das Bundesverfassungsgericht in der Solange I-Entscheidung die umfassende Prüfung von Gemeinschaftsrecht am Maßstab der Grundrechte des Grundgesetzes vorbehalten, „solange der Integrationsprozess der Gemeinschaft nicht so weit fortgeschritten ist, dass das Gemeinschaftsrecht auch einen von einem Parlament beschlosse-

1 BVerfGE 37, S. $271 \mathrm{ff}$.

2 EuGH, Rs. 6/64, Slg. 1964, 1251. 